\title{
Need for an oncological audit service
}

\author{
Adrian Pablo Hunis ${ }^{1,2 *}$ \\ ${ }^{1}$ University of Buenos Aires, Argentina \\ ${ }^{2}$ Universidad Maimónides, Argentina
}

\section{Introduction}

Spending on cancer drugs has almost doubled in the last five years and the increase continues to grow, imposing a substantial financial burden on cancer patients and their Health Systems. Only doctors trained in modern cancer treatment are able to evaluate, within a scientific and academic context, which patient should be treated, and which patient should enter a palliative care program.

An AUDITOR IN ONCOLOGY must prove to be a specialist in oncology, have previous experience and handle concepts related to indications of high cost studies, genetic determinations, biomarkers, molecular biology, diagnostic imaging, indications of surgical practices, radiant and obviously, medical- oncological

The great "secret" of good audit work is the valuation of the equation "COST-EFFECTIVE" or "COST-INEFFECTIVE" and demonstrate it with bibliographic information based on scientific evidence.

A well-founded rejection of oncological treatment pays more than the auditor's annual salary, generates savings for the Health System and allows the use of this resource for a cause that deserves it.

\section{Considerations}

One of the main drivers of this economic growth are drugs directed against cancer: small molecules, monoclonal antibodies, antiangiogenesis, vaccines and other cancer therapies that target specific genomic aberrations.

For the exaggerated expense that these drugs cause, there are authors who already talk about "FINANCIAL TOXICITY"

The financial burden resulting from a cancer diagnosis and/or treatment is referred to as financial toxicity, although the exact definition differs between studies. It is generally recognized that patients with a history of cancer experience greater out-of-pocket (OOP) costs and are more likely to report being unable to work or have more missed work days than individuals without a history of cancer. These OOP costs are expected to increase as more oral oncology drugs are approved because the patient's pharmacy coinsurance is typically higher than the costsharing requirement for infused therapies.

Numerous studies have attempted to quantify the financial toxicity associated with cancer. Though it is clear that cancer is associated with an extreme financial burden, the extent of the burden varies widely depending on study design and measurement parameters, type of treatment, and type of cancer. Certain factors are consistently associated with greater financial toxicity, including younger age at diagnosis, lower income at baseline, nonmarried status, and public insurance.

\section{Financial burden during treatment}

A systematic review of 45 studies published between 1990 and 2015 found that mean annual productivity loss was $\$ 380$ to $\$ 8236$. Financial distress of some kind was reported by $47 \%$ to $49 \%$ of patients. Another systematic review that included 25 studies published between 2013 and 2016 found that $28 \%$ to $48 \%$ of patients reported financial toxicity. The researchers found that younger age, female gender, low income at baseline, adjuvant treatment, and a more recent diagnosis were associated with financial toxicity.

A large study of 19.6 million cancer survivors from the 2011 Medical Expenditure Panel Survey found that $28.7 \%$ of cancer survivors reported financial burden, and financial burden was associated with lower physical and mental functioning scores, as well as depressed mood and concern for cancer recurrence. In a survey, $46 \%$ of adults who had undergone a stem cell transplant reported a decrease in income following the transplant and 56\% reported some type of financial hardship, including difficulty paying monthly bills, not having sufficient funds at the end of the month, and/or dissatisfaction with their present finances.

Some data suggest that younger patients can overcome financial hardships caused by a cancer diagnosis. A narrative systematic review of 23 studies found that education and employment were often delayed for patients diagnosed with cancer as an adolescent or young adult, but the researchers determined the individuals who had survived cancer eventually achieved careers as successful as those of the healthy controls.

\section{Debt and bankruptcy during treatment}

The extent of debt accrual or the need to file for bankruptcy due to treatment costs varies widely, but overall, bankruptcy rates among survivors of cancer are low. A systematic review of 45 studies reported that $12 \%$ to $62 \%$ of patients reported being in debt due to treatment. A survey of 4719 cancer survivors aged 18 to 64 years found that $33 \%$ had accrued debt and 3\% filed for bankruptcy; of those who accrued debt, $55 \%$ had at least $\$ 10,000$ of debt.

A longitudinal cohort study of patients aged 50 and older that included 9.5 million new cancer diagnoses between

${ }^{\star}$ Correspondence to: Adrian Pablo Hunis, MD, Board Certified in Clinical Oncology (AAOC; AMA; ANM), Assistant Professor of Internal Medicine (UBA), Director of the Career of Medical Specialist in Oncology (UBA), Head Professor of the Oncology (U. Maimónides), Argentina, E-mail: ahunis@fmed.uba.ar; aphunis@gmail.com

Received: July 02, 2018; Accepted: July 20, 2018; Published: July 23, 2018 
2000 and 2012 found that $42.4 \%$ of patients had used all of their financial assets 2 years after diagnosis, with financial insolvency persisting among $38.2 \%$ after 4 years. Factors associated with financial toxicity included worsening cancer, need for continued treatment, income, female sex, and having Medicaid or uninsured status.

\section{Effects on treatment}

Financial toxicity can affect treatment adherence, as patients may skip treatments or visits to reduce costs. In a systematic review, $4 \%$ to $45 \%$ of patients reported not adhering to treatment due to cost. Copay assistance is frequently available from drug manufacturers for patients with private insurance, but not for patients with Medicare because of federal regulations.

This financial assistance can dramatically reduce drug costs, but the copay cards typically expire after a period of time. Other OOP costs accrue as well, such as transportation, hospital or provider bills, and lost work days.

\section{Financial outcomes of cancer survivors}

In a cohort study, childhood cancer survivors were more likely than their siblings to have greater OOP medical costs that were at least $10 \%$ of their annual income, and a higher percentage of income spent on OOP costs was associated with difficulty paying medical bills; deferring health care, treatment, tests, or follow-up visits; and considering filing for bankruptcy.

In a cohort of 6012 women from the Life and Longevity After Cancer ancillary study of the Women's Health Initiative, $6 \%$ of women experienced some financial burden during the mean 9.2 years after a cancer diagnosis.

Significant debt, including filing for bankruptcy, occurred among $1.8 \%$ of women. Factors associated with financial burden included younger age, lower household income, shorter time since diagnosis, African-American race, lack of private health insurance, presence of comorbidities, treatment with chemotherapy, and regional stage of disease at diagnosis.

Financial toxicity is a potential consequence of cancer treatment, particularly among patients diagnosed at an earlier age and those of lower socioeconomic status or who have public health insurance. Importantly, financial toxicity can affect treatment because patients may not adhere to treatment in an effort to lower their OOP costs. Clinicians should be aware of the challenges that patients face in relation to financial toxicity and set up the appropriate processes and procedures to discuss financial factors that are relevant to each patient's treatment plan.

Currently, a group led by the Abramson Cancer Center of the University of Pennsylvania has proposed three solutions to maximize the clinical benefit and affordability of anti-cancer drugs using state-ofthe-art drugs, which shows that this problem is not It is only from the "emerging" countries, if not from all over the world.

The lead author is Justin E. Bekelman, MD, associate professor of Oncology and a member of Penn's Abramson Cancer Center.

To consider possible solutions, the University of Pennsylvania convened the Gant Consortium, a multidisciplinary group of experts and interested parties in cancer medicine, patient advocacy, pharmaceutical and insurance companies, and economics, health policies, laws and regulations, co-chaired by Bekelman.
On the basis of these discussions, the authors propose three main solutions:

1. The United States Food and Drug Administration (FDA) must develop a guide that defines the minimum sizes of clinically significant effects needed for the approval of targeted drugs against cancer.

2. The Centers for Medicare and Medicaid Services (CMS) must conduct a demonstration project in which Medicare negotiates the prices of cancer drugs and use form tools to prioritize highly effective drugs while protecting access to the drugs. treatment for each indication of cancer.

3. Treatment guidelines should prioritize anti-cancer drugs and treatment regimens based on clinical benefit and price.

The remarkable national commitment to cancer research and the promise, expense and projected growth of cancer drugs require solutions to promote medicines that produce significant clinical benefits while reducing overall price growth and out-of-pocket expenses from the patients.

Spending on cancer drugs in the US it increased from $\$ 26$ billion in 2012 to more than $\$ 45$ billion in 2016 , and 60 percent of that growth is the result of spending on next-generation drugs aimed at cancer.

According to at least one calculation, anti-cancer drugs will represent a quarter of the US advanced pharmaceutical research and development portfolio. By the year 2021, and 87 percent of these products will be specific agents.

The authors point to two specific medications as examples.

The first is imatinib, a tyrosine kinase inhibitor used to treat chronic myeloid leukemia (CML).

It is highly effective and has a relatively low toxicity compared to previous CML therapies.

The overall 10-year survival rate for patients with CML who take the medication is 84 percent, and the introduction of the drug in 2001 reduced the mortality rate of CML cases by more than half.

US In 2015, the estimated monthly Medicare price for the drug was $\$ 9,299$. By contrast, the authors also consider neratinib, another tyrosine kinase inhibitor in patients with early-stage breast cancer. The drug was approved by the FDA after improving the survival rate free of invasive disease by 2 percent (from 92 percent to 94 percent) after two years of follow-up, but without published survival data.

The estimated monthly price of the drug is $\$ 10,500$. "Imatinib shows the promise of targeted therapy, while neratinib exemplifies the concern that marginally effective treatments are putting pressure on health care expenditures in the United States," he said. Lead author Steven Joffe, MD, MPH, Emanuel and Robert Hart, professor of medical ethics and health Policy and co-chair of the Gant Consortium.

"Distorted prices of ineffective cancer drugs could displace the capacity of the US health system." To pay for highly effective anticancer drugs that produce significant clinical benefits or for other therapies of public health importance. "The FDA's regular approval for drugs is based on the demonstration of clinical benefit, defined as a longer, better life. quality of life or a substitute established for one of the two. 
The accelerated approval of the FDA for medicame These are based on reasonably likely results to predict clinical benefit. "The FDA has not specified what characterizes the significant clinical benefit for regulatory approval," Bekelman said. "This ambiguity is problematic in cases where new drugs directed against cancer show statistically significant improvements, but clinically questionable.

A thoughtful guide on what defines the minimum clinical benefit for drug approval would help manufacturers design appropriate trials and help patients and their doctors make decisions about cancer therapies. "The researchers also pointed out the need for Medicare can negotiate drugs for cancer, something that is currently not allowed.

From now on, Medicare pays for cancer drugs with the benefits of Part B and D. In Part B, hospitals and doctors buy the drug and then bill Medicare 6 percent above average selling prices. According to Part $\mathrm{D}$, insurance companies or pharmaceutical benefit managers generally administer price negotiations.

"As the largest purchaser of anti-cancer drugs in the USA in the US, Medicare must test a program where it has the tools to negotiate directly with the drug manufacturers on the price", said Joffe. This can be achieved while providing patients with access to anti-cancer drugs. for each indication available. "Finally, the authors call for evidencebased treatment guidelines to give physicians and patients better ability to consider the prices of targeted cancer drugs along with their benefits and harms when selecting treatments. While the organizations that develop the treatment, guidelines have taken steps to incorporate the costs, they should go further".

"The guidelines should categorize cancer regimens and cancer drugs that are driven by effectiveness and price and promote greater transparency of treatment prices" [1-11].

\section{References}

1. Financial Toxicity and Cancer Treatment (PDQ)-Health Professional Version. National Cancer Institute. Updated January 30, 2018. https://www.cancer.gov/aboutcancer/managing-care/track-care-costs/financial-toxicity-hp-pdq. Accessed July 20, 2018.

2. Altice CK, Banegas MP, Tucker-Seeley RD, Yabroff KR (2016) Financial Hardships Experienced by Cancer Survivors: A Systematic Review. J Natl Cancer Inst 109. [Crossref]

3. Gordon LG, Merollini KMD, Lowe A, Chan RJ (2017) A Systematic Review of Financial Toxicity Among Cancer Survivors: We Can't Pay the Co-Pay. Patient 10: 295-309. [Crossref]

4. Kale HP, Carroll NV (2016) Self-reported financial burden of cancer care and its effect on physical and mental health-related quality of life among US cancer survivors. Cancer 122: 283-289. [Crossref]

5. Abel GA, Albelda R, Khera N, Hahn T, Salas Coronado DY, et al. (2016) Financial hardship and patient-reported outcomes after hematopoietic cell transplantation. Biol Blood Marrow Transplant 22:1504-1510. [Crossref]

6. Stone DS, Ganz PA, Pavlish C, Robbins WA (2017) Young adult cancer survivors and work: a systematic review. J Cancer Surviv 11: 765-781. [Crossref]

7. Banegas MP, Guy GP Jr, de Moor JS, Ekwueme DU, Virgo KS, et al. (2016) For working-age cancer survivors, medical debt and bankruptcy create financial hardships. Health Aff (Millwood) 35: 54-61. [Crossref]

8. Gilligan AM, Alberts DS, Roe DJ, Skrepnek GH (2018) Death or debt? National estimates of financial toxicity in persons with newly-diagnosed cancers. Am J Med. [Crossref]

9. Fonseca R, Hinkel J (2018) Value and cost of myeloma therapy — we can afford it. $A m$ Soc Clin Oncol Educ Book 38: 647-655.

10. Nipp RD, Kirchhoff AC, Fair D, Rabin J, Hyland KA, et al. (2017) Financial Burden in Survivors of Childhood Cancer: A Report from the Childhood Cancer Survivor Study. $J$ Clin Oncol 35: 3474-3481. [Crossref]

11. Hastert TA, Young GS, Pennell ML, Padamsee T, Zafar SY, et al. (2018) Financial burden among older, long-term cancer survivors: Results from the LILAC study. Cancer Med. [Crossref]

Copyright: (C2018 Hunis AP. This is an open-access article distributed under the terms of the Creative Commons Attribution License, which permits unrestricted use, distribution, and reproduction in any medium, provided the original author and source are credited. 